\title{
Hindsight bias: An interaction of automatic and motivational factors?
}

\author{
WOLFGANG HELL, GERD GIGERENZER, SIEGFRIED GAUGGEL, \\ MARIA MALL, and MICHAEL MÜLLER \\ University of Constance, Constance, Federal Republic of Germany
}

\begin{abstract}
If subjects are asked to recollect a former response after having been informed about the correct response, their recollection tends to approach the correct response. This effect has been termed hindsight bias. We studied hindsight bias in an experiment requiring numerical responses to almanac-type questions for physical quantities. We varied (1) the time at which the correct information was provided, (2) the encoding of the original responses by asking/not asking subjects to give a reason for the respective response, and (3) the motivation to recall correctly. We found that hindsight is less biased if reasons are given and if the correct information is provided at an earlier time. Motivation had only interactive effects: (1) With high motivation to recall correctly, the time the correct information was provided had no influence. (2) With reasons given, the variation of motivation showed no effect. These results rule out purely motivational and purely automatic explanations.
\end{abstract}

Subjects who are asked almanac-type questions and are given the correct information at a later time have been found to "correct" their memory for their original responses in the direction of the correct response (e.g., Fischhoff, 1977). This result has been termed distorted or biased hindsight. Hindsight bias has been demonstrated for several types of knowledge: football game results (Leary, 1981), elections (Leary, 1982; Synodinos, 1986), political events (Fischhoff \& Beyth, 1975), medical diagnoses (Arkes, Wortmann, Saville, \& Harkness, 1981), and general knowledge (Fischhoff, 1977; Wood, 1978). In addition to its wide-ranging effect, hindsight bias has been found to be surprisingly resistant to experimental modifications. It has been shown to be unaffected by telling people to work hard (Fischhoff, 1977), by informing subjects about this bias (Fischhoff, 1977), and by manipulating the perspective of the subjects (tell as you told/tell as other students would tell; Wood, 1978). Hindsight has been found to be less biased with fewer repetitions of the correct information (Wood, 1978), with a smaller time delay between the event and the recollection of the past prediction (Fischhoff \& Beyth, 1975), and for the a priori more probable outcome (Arkes et al., 1981). Only one manipulation has been found to eliminate the bias: telling the subjects that the "correct" information already incorporated into the memory was ("sorry about that") actually wrong (Hasher, Attig, \& Alba, 1981).

Two classes of theories, motivational and cognitive, have been proposed to account for the hindsight bias phenomenon. On the one hand, hindsight bias can be conceived as stemming from esteem-maintaining motives that

Address correspondence to Wolfgang Hell, Fachbereich Psychologie, Universität Münster, Fliednerstrasse 21, $\mathbf{4 4 0 0}$ Münster, Federal Republic of Germany. lead the subject to try to show himself/herself as being more knowledgeable. According to this class of theories, the amount of the bias should be influenced by subjects' motivation and strategies; that is, it should be, to a certain extent, under the control of the subject (e.g., Campbell \& Tesser, 1983). On the other hand, hindsight can be regarded as an automatic process linked to the usual laws of information processing and storage, making the bias unavoidable by the subject. The assimilation idea is a well-known example: "Upon receipt of outcome knowledge judges immediately assimilate it with what they already know about the event in question" (Fischhoff, 1975 , p. 297). The support for the motivational view has been weak. Leary $(1981,1982)$ found no effect of a low or a high level of ego-involvement or of mode of prediction (public vs. anonymous). He argued, therefore, that self-esteem and self-presentation concerns played no role in the hindsight distortion. The above-mentioned "workhard" condition of Fischhoff (1977), which failed to show any selective effect upon the amount of the hindsight bias, also can be counted as a motivational manipulation. Synodinos (1986) recently found no effect of global selfesteem and political involvement on hindsight bias for the prediction of an electoral contest in Hawaii. Campbell and Tesser (1983) are the only authors we know of who reported a motivational influence. In their subjects, dogmatism, intolerance for ambiguity, social desirability, and self-reported ego-involvement were positively correlated with the amount of hindsight bias in the standard almanactype questions procedure.

Most authors, however, have argued that hindsight bias is automatic, that is, unconscious and unavoidable by the subject, a by-product of the normal way of information processing and storage. The notable exception to this notion is the above-mentioned demonstration by Hasher 
et al. (1981) that the original memory is completely recoverable if the subject is led to believe that the recent information was wrong. In no way can this finding be reconciled with any conception that postulates automatic assimilation of memories into a single memory trace. In general, however, hindsight bias has been successfully explained by postulating imperfect memory recall due to updating and erasing of stored information and due to the influence of contextual information (Bartlett, 1932). Our experiment that uses the usual almanac-type questions hindsight format has two aims: first, to study the joint influence of two variables known to have an impact upon the quality of memory-that is, depth of encoding and retention time-and, second, to investigate the influence of one motivational variable-that is, incentive to recall correctly.

In hindsight experiments, depth of encoding of the original opinion has not been explicitly manipulated so far. Retention time has been varied by Fischhoff and Beyth (1975), with the reported result that hindsight bias was larger with a longer time between the prediction and the recollection of the prediction. However, these data were collected in a between-subjects design in which retention time was completely confounded with class level of the subjects (e.g., advanced methodology class vs. introductory psychology class). Three different but interdependent retention times can be differentiated: (1) the time between the original response of the subject to a question and the presentation of the correct information; (2) the time between the presentation of the correct information and the recollection, that is, the response to the task of recollecting the original response; and (3) the sum of both, the time between the original response and the recollection. In our experiment, the latter time was held constant while the other two were varied in a within-subjects design. Orthogonally to this variation, the depth of encoding was varied as well.

What are the predictions for the result of these two manipulations thought to influence retention, disregarding for the moment the motivational variable? As for depth of encoding, the prediction is clear-cut. From memory research, there is ample evidence that depth of encoding enhances recall (e.g., Craik \& Lockhart, 1972; Craik \& Tulving, 1975). In hindsight bias research, one implicit manipulation of strength of encoding is known to us. Wood (1978) found hindsight to be more biased with more study trials for the correct information. In contrast, with a deeper encoding of the original response-that is, with a stronger and more readily accessible memory tracewe should find less hindsight bias.

The predictions for the manipulation of the time at which the correct information is given are less unequivocal. Conflicting information after the initial encoding has been shown in memory research to mislead the subjects (e.g., Loftus, 1975). This result, however, has not invariably found support and arguably depends upon minor procedural variables (Kroll \& Timourian, 1986; McCloskey \& Zaragoza, 1985). Snyder and Uranowitz
(1978) introduced new information by providing subjects a new perspective to a previously read story, either immediately after encoding or immediately before recall, and reported no influence of the time of this additional information. In our experiment, the same manipulation was used in a hindsight experiment; that is, the correct information was given either immediately after the original response or immediately before its recollection, with the time between the two responses being held constant. Schema theories that postulate a single holistic memory trace from different sources of information (see Alba \& Hasher, 1983, for a review) allow predictions if assumptions on the time course of the forming of a single trace are made. The "immediate assimilation" per se, postulated by Fischhoff (1975), does not consider a time-ofcorrect-information effect. For both times, the same amount of hindsight bias should show. If, however, the assimilation or forming of a single trace is assumed to be a gradual process that increases with a longer coexistence of two memory traces, then we should find less hindsight bias if the correct information is given immediately before recall, a condition in which both traces have coexisted for only a short time. A different assumption leads to the opposite prediction. Let the assimilation be immediate but its amount depend upon the relative trace strengths, and let the trace strengths decay with time. Then there should be more hindsight bias with a relatively stronger trace of the correct information, that is, if this information is given at a later time and therefore the trace for the orginal response is already weaker.

It should be noted, however, that there is one difference between hindsight experiments and memory experiments using conflicting information. In Loftus's (1975) experiments, for instance, in which details from a viewed traffic accident were requested, the questions were seeded with false information about the scene, putting pressure upon the subjects to update their memory with incoming conflicting information. Loftus and Loftus (1980) held that old information is updated and erased only if it is inconsistent with new information. In hindsight experiments, a subject might as well recall that she was told that the Eiffel tower is $300 \mathrm{~m}$ high as that she originally thought it was only $250 \mathrm{~m}$ high without experiencing any conflict comparable to that in Loftus's experiments.

In addition to and orthogonal to these manipulations of information processing variables, we also varied the motivation by giving a subgroup of the subjects a high incentive for correct recall of the original responses. On the one hand, this is a mere repetition of the try-hard manipulation by Fischhoff (1977) that had failed to reduce hindsight bias. However, with a higher incentive, an effect, if it exists, might be large enough to be found; that is, less hindsight bias might be found with a higher motivation to recall correctly. On the other hand, varying motivational and information processing variables in a single experiment allowed us to look for interactions that have not been studied previously. We had no specific idea which interaction to expect at the time of the onset of the ex- 
periment, but it is clear that any motivation-alone and any information-processing-alone theory cannot account for an interaction. Similarly, single or melted trace ideas leave no room for differential accessibility of traces, even with a high incentive. If, however, the memory trace for the original response is not melted together with the trace for the correct information-that is, if it is not inaccessible but merely weak, and therefore difficult to access-then the manipulation of the retrieval effort by manipulating the motivation should have an impact upon the amount of hindsight bias found.

For the dependent variable, we introduced a modification. We asked for numerical estimations for the responses to almanac-type questions, permitting a numerical estimate of the amount of hindsight bias. In most of the cited articles, subjects had to rate the probability that one particular response (of two) was correct. Hindsight bias, it has been argued, shows up in the deviation from the original rating found in the replication of the probability rating after the receipt of the correct information. We replaced subjective degree of belief in the truth of an assertion by estimation of physical quantities. No other study we know of has asked for a replication of numerical estimates of physical quantities by the same subjects.

In summary, memory trace strength, time of correct information, and motivation to recall correctly were independently varied in an almanac-type questions experiment, with numerical estimates as the dependent variable. Hindsight bias should be larger with weaker memory strength for the original response, there are contradictory predictions for the effect of the time of correct information, and a higher motivation to report correctly should be associated with less hindsight bias if the memory trace for the original response is accessible separately.

\section{METHOD}

Sixty students of a biology class of Constance University were paid DM 18 each for their participation. The data of 59 subjects were complete enough to be evaluated.

All subjects were tested together in a lecture room at two sessions, with an interval of 1 week. They were told that we planned to test general knowledge. At the first session, each student was given a booklet with 90 questions requiring numerical responses. The first 2 questions were warm-up questions. Only the remaining 88 questions were used for experimental purposes. For all of the questions, the subjects could be expected to have not exact knowledge, but a fair basis for a good guess. An example is " How long is a hundred-mark note?' The subjects were explicitly required to give an exact numerical estimate and not to respond, for example, "longer than $10 \mathrm{~cm}$ " or "10-20 cm." They had to use the unit given in the booklet ("cm" in the example cited). For half of the questions (reason-requested condition), the subjects had to write in a few words the reason for each estimate just below the response. Reasons given for responses to the above-mentioned question included "as long as two forefingers," "as long as my purse," and "visual memory representation." The reasons given were not evaluated. For the other half (no-reason-requested condition), no reason for the estimate was requested. Since the filling out was selfpaced, as were all further responses, we cannot know whether any effect of this manipulation is due to deeper semantic processing of the respective items or simply to longer exposure to them. After the completion of the first booklet, each subject received a new booklet with the correct answers to 44 of the questions, that is, to $50 \%$ of all questions excluding the warm-ups. To ensure the processing of the correct information, the subjects were requested to indicate for each correct answer whether or not their remembered response departed from the correct one by more than a factor of two. The data from this task were not evaluated.

One week later, the same subjects were given the same task with a third booklet that contained the correct answers to the remaining 44 questions and was otherwise identical to the second booklet. This was the manipulation of the second independent variable, time of correct information, that is, either immediately after the original response or immediately before the recollection. The time between original response and recollection was held constant at 1 week. This and the first variable, memory trace strength, were varied within subjects. The use of different booklets made sure that any individual question appeared with equal frequency in each of the experimental conditions. After filling out the third booklet, the subjects received the fourth and final booklet, which revealed the actual aim of the experiment. They were required to reproduce their original responses to the 88 questions, again giving a numerical value. The third independent variable, motivation, was varied between subjects in the following way: The high-incentive group, 28 of the evaluated subjects, were promised an additional fee of DM 25 if they were among the three best students, in terms of mean deviation from their original responses. This information was displayed at a prominent place in the final booklet, that is, after subjects had formed their memory traces for the original response and the correct information. The remaining 31 subjects, the low-motivation group (included, of course, in the set of possible "winners"), were not informed about the additional fee. After completion of the last booklet, the subjects were paid and received a leaflet indicating our intentions and main hypotheses.

The dependent variable, percentage amount of hindsight bias, was defined as $100(\mathrm{OR}-\mathrm{RC}) /(\mathrm{OR}-\mathrm{CI})$, with $\mathrm{OR}=$ numerical value of the original response, $\mathrm{RC}=$ numerical value of the recollection of it, and $\mathrm{Cl}=$ numerical value of the correct information. This index gives the percentage amount of hindsight bias, independent of the absolute numerical values of the unit used, and of the closeness of the original response to the correct one. When the subjects show the usual hindsight bias, this index has a value betwen $0 \%$ and $100 \%$, with higher values indicating more bias. The extreme values point to no hindsight bias $(0 \%)$ or complete hindsight bias $(100 \%)$. Values greater than $100 \%$ or less than $0 \%$ were, of course, not excluded from evaluation, but also were not expected to occur often. If, in the above-mentioned example, a subject guessed a 100 mark note to be $15 \mathrm{~cm}$ long, was given the information that it is $15.9 \mathrm{~cm}$ long, and remembered his/her original response as $15.3 \mathrm{~cm}$, the hindsight bias index is $33 \%$, indicating that this subject in this question "adapted" his/her memory by a third of the difference between the original and the correct information.

\section{RESULTS}

All hindsight bias indices were calculated, except for missing entries and cases of perfect knowledge (i.e., RC $=\mathrm{CI}$, for which the index is undefined ( $3.2 \%$ together). In $35.1 \%$ of all cases, the hindsight bias index was 0 ; that is, the subjects correctly recollected their original responses. For $30.9 \%$ of all responses, the index was greater than 0 and less than or equal to 100 . In $18.4 \%$ and $12.4 \%$ of all cases, respectively, the index was less than 0 or greater than 100 . The original idea, to calculate the means of the hindsight bias indices, proved to be nonsensical. Although by far the majority of the indices were 
found to be within the expected range, a few exorbitant values showed up. To use the above example, if the subject wrote $153 \mathrm{~cm}$ as the recollection of the original response, the index is calculated to be $15,333 \%$, due most probably to a writing error. Since we found several of these values, not all of which are as easy to explain as the above example, we decided not to discuss which data to discard for which reasons, but to use all of them and to perform all tests with the median values, unaffected by extreme data.

We determined the median value for each combination of conditions for each subject, a value based upon 22 entries, and used it as the respective datum for the analysis of variance with the within-subjects factors time of correct information and (no) reason requested, and the between-subjects factor motivation. Figure 1 shows the averaged medians. The effects in each of the $2 \times 2 \times 2$ cells are all positive; that is, there is no "negative" hindsight bias. The absolute size of effect, however, varies strongly with the condition, from only $1 \%$ to $22 \%$.

The (no)-reason-requested manipulation shows a significant main effect $[F(1,57)=19.3, p<.01]$. As can be seen in Figure 1, the effect is in the expected direction: more hindsight bias in the no-response-requested conditions (the upper four data points). Time of correct information was also found to be significant $[F(1,57)=$ $6.8, p<.05$ ], the bias being larger when the correct information was given at a later time (the four points at the right side in Figure 1). The influence of motivation was found in the expected direction, less hindsight bias with a higher incentive to recollect correctly, but it was statistically nonsignificant $[F(1,57)=2.5]$. The statistically significant interaction between time of correct information and motivation $[F(1,57)=6.1, p<.05]$ is evident from Figure 1. The effect of time of correct information is found only with no incentive to recall correctly and is completely canceled in the high-incentive group. The

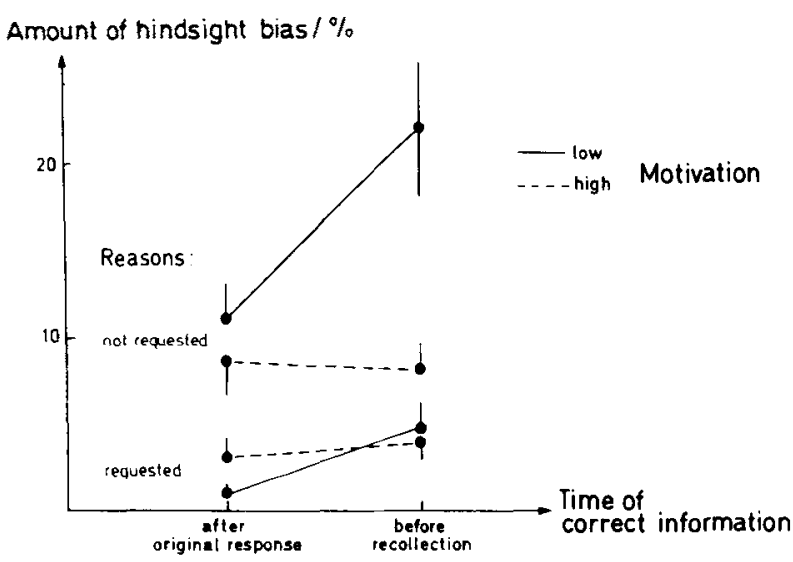

Figure 1. Amount of hindsight bias (averaged median values) as a function of (1) motivation (high/low incentive to recall correctly), (2) time of correct information (immediately after original response/immediately before recollection), and (3) reason for the original response (requested/not requested). Standard errors of the means are indicated. interaction between incentive and the (no)-reasonrequested manipulation, less evident from Figure 1 but also significant $[F(1,57)=4.4, p<.05]$, can be described as incentive's having an influence only if no reason was requested. These interactions indicate that the motivation (incentive) variable, although not showing a statistically significant influence per se, considerably influences the effect from the manipulation of the other two variables. The third interaction, between time of correct information and (no) reason requested, was not significant $[F(1,57)=.76]$; nor was the triple interaction $[F(1,57)=1.6]$.

\section{DISCUSSION}

The least controversial result is the finding that requesting the subjects to give reasons for the original response reduces the hindsight bias. This fits nicely with Wood's (1978) result that presenting the correct information more often leads to a larger hindsight bias. Both findings can be explained by the assumption that the (relative) trace strengths are involved in determining the amount of hindsight bias produced. A (deeper) memory trace for the correct information (more presentations) leads to a larger hindsight bias; a stronger trace for the original response, as supposedly attained by our reason-requested manipulation, leads to a smaller hindsight bias. A recent experiment, however, has failed to lead to any reduction of hindsight bias "by increasing foresight encoding elaboration" (Davies, 1987, p. 60). Davies asked subjects to read through descriptions of psychology experiments; half of them wrote their thoughts, especially with respect to reasons for possible outcomes, a manipulation thought to make the trace of the subjects' state of knowledge more durable. Two weeks later the subjects had to judge the a priori likelihood of the experimental outcomes, with or without outcome knowledge. In the notes condition, hindsight bias was not reduced. One major procedural difference that might account for the differing results was that the subjects in our experiment were asked to reproduce a given estimate, whereas the subjects in the outcome knowledge condition of Davies's (1987) experiment were asked to give estimates "as if they had been asked before they knew how the experiment turned out" (p. 55). Further research will show the generality of Davies's (1987) summary that "efforts to reduce hindsight bias should concentrate on postevent or postoutcome manipulations" (p. 64).

Relative trace strength also has been a concept used in the successful explanation of a possible time-of-correctinformation effect. If assimilation of memory traces (Fischhoff, 1975) is assumed to depend upon the relative strengths of memory traces, then more hindsight bias should occur with a relatively stronger trace of the correct information. If the time between the original response and the recollection is held constant, as in the present experiment, and the uncontroversial assumption of the 
decline of memory trace strength with time is introduced, then hindsight should be more biased with a later presentation of correct information, as was indeed found. The alternative predictions of either no time-of-correctinformation effect derived by assuming immediate assimilation or the opposite time-of-correct information effect derived by assuming assimilation that increases with time of coexistence of possible conflicting information independent of trace strength have been ruled out by the result. The successful prediction, however, can also be derived, following closely McCloskey and Zaragoza (1985), by assuming that the subject uses a mixture of correct recollections of the original responses, guesses guided by recollection of the correct information, and guesses without any recollection whatsoever. The finding that the original responses were correctly recollected in $35.1 \%$ of all cases gives support to McCloskey and Zaragoza's (1985) idea. The two nonmotivational manipulations, giving the correct information at different times and leading the subject to a different processing of the original response, had independent effects that showed no interaction. So far the picture is easy to understand. We have identified two variables that influence the amount of hindsight bias, that are independent of each other, and that can both be explained by introducing assumptions upon trace strengths that are in line with other findings from memory research.

Motivation per se, manipulated by giving (no/a high) incentive for correct recall, had no statistically significant effect. One can speculate that we might have found a significant influence had we only used the normal hindsight design (upper two curves in Figure 1) without the reason-requested condition or had we used a withinsubjects design for motivation as well. Support for the last conjecture is found in the fact that the time-of-correctinformation effect, which is statistically significant, has an almost identical mean effect as the nonsignificant motivation effect. But we can leave these questions to further experimentation and state that so far a high motivation, manipulated by an incentive (present experiment) or by the request to work hard (Fischhoff, 1977), has not been shown to reduce hindsight bias significantly.

Let us turn now to the more interesting interactions: that a high incentive to recall correctly cancels the timeof-information effect completely, and that the request to give a reason cancels the motivation effect completely. The ideas presented so far to explain the hindsight bias effect have either stressed information processing variables (e.g., Fischhoff, 1977) or motivational variables (e.g., Campbell \& Tesser, 1983), and researchers have tried to differentiate between these two lines of reasoning. We have not presented any predictions for interactions since we had none at the onset of the experiment; however, any model not using ideas from both domains is unable to explain the interactions found. In order to explain these interactions, we introduce the following modification of the model presented above. We assume that the subject is able to recover a relatively weak memory trace with a certain probability if he/she has a good reason to try hard (whether simply being told to try hard is a good reason to try hard is subject to doubt). For a stronger memory trace, the amount of effort used without incentive is high enough to prevent any considerable amount of hindsight bias. With a low motivation and a weak memory trace for the response (no reason requested) and a strong memory trace for the correct information (immediately before recollection), hindsight bias amounts to $22 \%$. The four no-reason-requested conditions, which correspond to the usual depth of encoding used by other authors, lead to $12 \%$ hindsight bias on the average, whereas requesting reasons reduces hindsight bias to a mere $3 \%$. We can, therefore, safely conclude that a prerequisite for hindsight bias to occur is a weak memory trace for the original response. If this condition is met, the amount of hindsight bias found depends upon the relative memory trace strengths, with the exception of those subjects who are motivated to recover even weak traces. Hindsight bias, therefore, is not automatic in the sense of being unavoidable. Hasher et al.'s (1981) finding that subjects can retrieve the original trace if they are led to believe that the "correct information" was wrong has found corroboration.

The amount of hindsight bias found in the present study cannot directly be compared with the numerical results from usual experiments using two-alternative questions and confidence ratings. Hindsight bias in these experiments is argued to occur if the mean number of correctly recalled confidence ratings decreases and, at the same time, the mean confidence ratings for correct alternatives increases. Fischhoff (1977, Experiment 1), for instance, has found that in the hindsight group the mean number of correctly recalled confidence ratings was $13 \%$ lower than in a control group, and that in $72 \%$ of the incorrectly recalled confidence ratings a higher likelihood was assigned to the correct alternative. The only publication we know of that allows direct comparison is by Synodinos (1986), who asked subjects to predict (postdict) percentages of votes for three candidates in a gubernatorial election. With this small database, three data points, and keeping in mind that different subjects participated in the prediction and postdiction conditions, we can calculate the hindsight bias index with these data to be $21 \%$ on the average. Since the "correct information" was the actual result of the election, we can assume that the "original response," that is, the prediction before the election, had a relatively weak memory trace. Also, his subjects had no high incentive to give correct recollections. The comparable condition from our experiment, therefore, is the weak-trace low-motivation condition, which yielded an average percentage hindsight bias of $17 \%$, quite close to the value calculated from Synodinos's data.

That we have found a motivational influence and others have not (Fischhoff, 1977; Leary, 1981, 1982; Synodinos, 1986) is not surprising for two reasons. First, we found motivation to interact with other variables, and the other researchers and we failed to find a motivational influence 
per se. Second, the label "motivation" is host to very different operationalizations. Only Fischhoff's (1977) tryhard condition is comparable to our monetary-incentive condition, both failing to have a main effect on hindsight bias. Campbell and Tesser (1983), the only authors to report a motivational influence so far, found a correlation between the amount of hindsight bias and esteemmaintaining variables, such as social desirability and egoinvolvement.

We have tried to explain the interactions of the motivational variable with the information-processing variables by assuming that an incentive leads subjects to use more effort to recover even the weak memory traces. This idea is not new to memory research. At the latest, since the introduction of the signal detection model into memory research (Murdock, 1965; Lockhart \& Murdock, 1970), the notion of all-or-none recoverability of memory contents is obsolete. According to the signal detection model, the subject shifts his/her criterion with the experimental demands. The criterion shift idea has also been used, for example, to explain the alleged hypnotic memory enhancement (Smith, 1983). On the other hand, Loftus (1979) reported that a monetary incentive failed to improve her subjects' recollections. The difference between our task and Loftus's was that in her experiment only one of two traffic signs could possibly have been seen, whereas our subjects had no objective reason to suppress one of the memory traces.

\section{REFERENCES}

Alba, J. W., \& HASher, L. (1983). Is memory schematic? Psychological Bulletin, 93, 203-231.

arkes, H. R., Wortmann, R. L., Saville, P. D., \& Harkness, A. R. (1981). Hindsight bias among physicians weighing the likelihood of diagnoses. Journal of Applied Psychology, 66, 252-254.

BARTLETT, F. C. (1932). Remembering: $A$ study in experimental and social psychology. Cambridge: Cambridge University Press.

CAmpBell, J. D., \& Tesser, A. (1983). Motivational interpretations of hindsight bias: An individual difference analysis. Joumal of Personality, 51, 605-620.

Craik, F. I. M., \& Lockhart, R. S. (1972). Levels of processing: A framework for memory research. Journal of Verbal Learning \& Verbal Behavior, 11, 671-684.

Craik, F. I. M., \& Tulving, E. (1975). Depth of processing and the retention of words in episodic memory. Journal of Experimental Psychology: General, 104, 268-294.

DAviEs, M. F. (1987). Reduction of hindsight bias by restoration of foresight perspective: Effectiveness of foresight-encoding and hindsight-retrieval strategies. Organizational Behavior \& Human Decision Processes, 40, 50-68.

FischноF, B. (1975). Hindsight $\neq$ Foresight: The effect of outcome knowledge on judgment under uncertainty. Joumal of Experimental Psychology: Human Perception \& Performance, 1, 288-299.

FischHOFF, B. (1977). Perceived informativeness of facts. Journal of Experimental Psychology: Human Perception \& Performance, 3, 349-358.

FischHOFF, B., \& BeYTH, R. (1975). "I knew it would happen." Remembered probabilities of once-future things. Organizational Behavior \& Human Performance, 13, 1-16.

HASher, L., Attig, M. S., \& AlBa, J. W. (1981). I knew it all along: Or, did I? Journal of Verbal Learning \& Verbal Behavior, 20, 86-96.

Kroll, N. E. A., \& Timourian, D. A. (1986). Misleading questions and the retrieval of the irretrievable. Bulletin of the Psychonomic Society, 24, 165-168.

LEARY, M. R. (1981). The distorted nature of hindsight. Journal of Social Psychology, 115, 25-29.

LEARY, M. R, (1982). Hindsight distortion and the 1980 presidential election. Personality \& Social Psychology Bulletin, 8, 257-263.

LOCKHART, R. S., \& MURDOCK, B. B., JR. (1970). Memory and the theory of signal detection. Psychological Bulletin, 74, 100-109.

LoFTUS, E. F. (1975). Leading questions and the eyewitness report. Cognitive Psychology, 7, 560-572.

LofTus, E. F. (1979). Eyewitness testimony. Cambridge, MA: Harvard University Press.

LofTUs, E. F., LofTus, G. R. (1980). On the permanence of stored information in the human brain. American Psychologist, 35, 409-420.

McCloskeY, M., \& ZARAGoZA, M. (1985). Misleading postevent information and memory for events: Arguments and evidence against memory impairment hypotheses. Journal of Experimental Psychology: General, 114, 1-16.

MuRDOCK, B. B., JR. (1965). Signal-detection theory and short-term memory. Journal of Experimental Psychology, 70, 443-447.

SmITH, M. C. (1983). Hypnotic memory enhancement of witnesses: Does it work? Psychological Bulletin, 94, 387-407.

SNYDER, M., \& URANOWITZ, S. W. (1978). Reconstructing the past: Some cognitive consequences of person perception. Journal of Personality \& Social Psychology, 36, 941-950.

Synodinos, N. E. (1986). Hindsight distortion: "I knew it all along and I was sure about it." Journal of Applied Social Psychology, 16, 107-117.

Wood, G. (1978). The knew-it-all-along effect. Journal of Experimental Psychology: Human Perception \& Performance, 4, 345-353.

(Manuscript received May 29, 1987; revision accepted for publication January 28, 1988.) 